\title{
Radioimmunoassay Studies of Human Apolipoprotein E
}

\author{
Conrad B. Blum, Lieselotte Aron, and Robert Sciacca, \\ Arteriosclerosis Research Center, and Department of Medicine, \\ Columbia University, College of Physicians \& Surgeons, New York 10032
}

A B S T R A C T This report describes the development and first applications of a sensitive and specific double antibody radioimmunoassay for human apolipoprotein $\mathrm{E}(\mathrm{apoE})$. ApoE was purified from the very low density lipoproteins of hypertriglyceridemic patients by heparin-agarose affinity chromatography, DEAE-cellulose chromatography, and preparative polyacrylamide gel electrophoresis. The purified apoprotein had an amino acid composition characteristic of apoE and resulted in the production of monospecific antisera when injected into rabbits. The radioimmunoassay, which was carried out in the presence of $5 \mathrm{mM}$ sodium decyl sulfate, had a working range of 0.8-12 ng. The withinassay coefficient of variation was $9 \%$ and the coefficient of variation for systematic between-assay variability was $3 \%$. Prior delipidation of samples with organic solvents did not alter their immunoreactivity.

In 26 normal volunteers, the mean plasma apoE concentration was $36 \pm 13 \mu \mathrm{g} / \mathrm{ml}$. Hyperlipidemic patients $(n=68)$ had higher mean apoE levels. A single patient with type III hyperlipoproteinemia had a plasma apoE level of $664 \mu \mathrm{g} / \mathrm{ml}$. The plasma apoE level was independently related to plasma cholesterol and triglyceride levels in a population of 108 normal and nonchylomicronemic hyperlipidemic patients. The multiple correlation coefficient for this relationship was 0.73 . Thus, variation in plasma cholesterol and triglyceride concentrations described $53 \%$ of the variation in apoE concentrations in this population.

The lipoprotein distribution of apoE was investigated by agarose column chromatography and ultracentrifugation of plasma. Agarose column chromatography demonstrated that all or nearly all plasma apoE is associated with lipoproteins. In plasma from normal volunteers and hypercholesterolemic patients, apoE was found in two discrete lipoprotein classes: very low density lipoproteins and a set of lipoprotein particles with size and density characteristics similar to $\mathrm{HDL}_{2}$. In hypertriglyceridemic patients, nearly all apoE was associated with the triglyceride-rich lipoproteins.

Received for publication 14 March 1980 and in revised form 28 Iuly 1980.

\section{INTRODUCTION}

Apolipoprotein E (apoE), ${ }^{1}$ also known as the argininerich apolipoprotein, is a glycoprotein with a molecular weight of $\sim 35,000$ and has been reported to be an important constituent of normal human very low density lipoproteins (VLDL) (1-4). This apoprotein, as well as apoB of low density lipoproteins (LDL), interacts with the cell surface receptors for LDL, thereby delivering cholesterol to cells $(5,6)$. Furthermore, it is a major component of lipoproteins induced by cholesterol feeding in several species including humans (710). These cholesterol-induced lipoproteins include those that have been designated as $\mathrm{HDL}_{\mathrm{c}}$ by Mahley et al. (7-9). Electrophoresis of the apoproteins of human high density lipoprotein (HDL) suggests that apoE is a very minor component of normal human HDL, but the portion of the total plasma apoE content that is contained in the HDL density range has not been established.

The concentration of apoE in normal human plasma has been measured by radial immunodiffusion (11), by electroimmunoassay (12), and most recently by radioimmunoassay (13). The apoE content of VLDL has been assayed by quantitative densitometry of stained polyacrylamide gels containing the electrophoresed apoproteins of VLDL (14). In normal VLDL, apoE was found to account for a mean of $13 \%$ of the total protein present and $21 \%$ of the non-B apoproteins. Furthermore, the apoE content of VLDL was shown to vary with lipoprotein particle size, suggesting that apoE is removed from VLDL particles as they undergo catabolism. The metabolic fate of apoE from catabolized VLDL is, however, not known. Any plasma fractions other than VLDL which normally contain apoE would be candidates as acceptors for apoE from triglyceriderich lipoproteins.

The objectives of the present studies were to de-

\footnotetext{
${ }^{1}$ Abbreviations used in this paper: $\mathrm{ApoE}$, apolipoprotein E; BSA, bovine serum albumin; DTT, dithiothreitol; HDL, high density lipoprotein; LDL, low density lipoprotein; PBS, phosphate-buffered saline; SDS, sodium dodecyl sulfate; VLDL, very low density lipoprotein.
} 
termine the lipoprotein distribution of apoE in normal and hyperlipidemic humans and to determine what relationships may exist between plasma apoE levels and fasting plasma lipid levels. To pursue these objectives we have developed a sensitive and specific radioimmunoassay for human apoE. This report describes the development of this assay and its first applications in clinical investigation. Using this assay, the levels of apoE in plasma were determined in a series of normal subjects and hyperlipidemic patients. The distribution of plasma apoE among the plasma lipoproteins was also determined for both normal and hyperlipidemic persons. Some of the findings presented here have been reported in abstract form (15).

\section{METHODS}

\section{Subjects}

Normal volunteers comprised 26 persons taking no medications who were selected without prior knowledge of their plasma lipid levels. They ranged in age from 22 to $62 \mathrm{yr}$. The 89 hyperlipidemic patients were all being followed in the Lipid Clinic of the Columbia University Specialized Center of Research in Arteriosclerosis. All patients had normal renal, hepatic, and thyroid function; all but five had normal levels of fasting serum glucose. The five diabetics included one patient identified as diabetic in Table III, two patients with fasting chylomicronemia and fasting plasma triglycerides $>1,000 \mathrm{mg} / \mathrm{dl}$, and two patients with plasma triglycerides of 389 and $686 \mathrm{mg} / \mathrm{dl}$ but plasma cholesterol concentrations $<275 \mathrm{mg} / \mathrm{dl}$. 79 patients were taking no medication; 10 were being treated with hypolipidemic agents ( 7 with cholestyramine or colestipol, 2 with clofibrate, and 1 with nicotinic acid). None of the patients with plasma triglycerides $>1,000$ $\mathrm{mg} / \mathrm{dl}$ were being treated with a hypolipidemic agent when sampled for this study.

Blood samples were obtained after an overnight fast. Venous blood was collected with EDTA $(1 \mathrm{mg} / \mathrm{ml})$ as anticoagulant, and was stored at $4^{\circ} \mathrm{C}$. Plasma was separated within $4 \mathrm{~h}$ of the time of blood collection. Plasma was stored at $-20^{\circ} \mathrm{C}$ for up to 8 mo before assay of apoE.

\section{Isolation of apoE}

ApoE was isolated from the VLDL of pooled plasma of hypertriglyceridemic patients (Fig. 1). Venous blood from fasting patients was collected in tubes containing solid EDTA $(1 \mathrm{mg} / \mathrm{ml})$ and was immediately chilled to $4^{\circ} \mathrm{C}$. Plasma was separated by centrifugation at $2,500 \mathrm{~g}$ for $20 \mathrm{~min}$, and VLDL was isolated by tube slicing after a single 18-h ultracentrifugation of the plasma at $60,000 \mathrm{rpm}$ at $4^{\circ} \mathrm{C}$ in a Beckman 70Ti rotor (Beckman Instruments, Palo Alto, Calif.) (16). The VLDL was dialyzed, lyophilized, and delipidated as previously described for HDL (17). VLDL apoproteins were then dissolved in $5 \mathrm{M}$ urea, $2 \mathrm{mM}$ sodium phosphate, $1 \mathrm{mg} / \mathrm{ml}$ dithiothreitol (DTT), $0.01 \%$ sodium azide pH 7.4 (urea-phosphate buffer with DTT) (Fig. 1, gel A).

Heparin-agarose affinity chromatography of the dissolved VLDL apoproteins was performed using a modification of the procedure of Shelburne and Quarfordt (18). $20 \mathrm{ml}$ of Sepharose CL4B, to which was bound $\sim 200 \mathrm{mg}$ of heparin (Fisher Scientific Co., Pittsburgh, Pa.) (19), was equilibrated with urea-phosphate buffer and packed into a $50-\mathrm{ml}$ plastic syringe that served as a column. The apoproteins (200-500 mg)

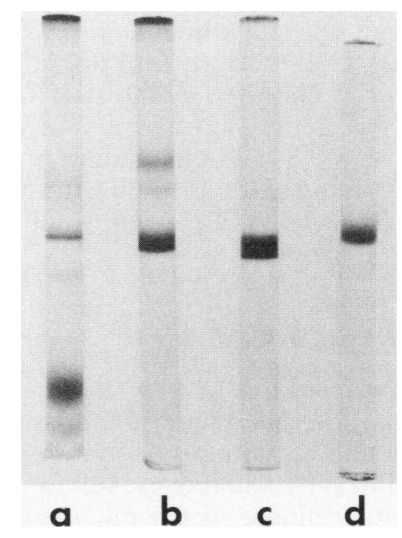

FIGURE 1 SDS polyacrylamide gel electrophoresis of apoE in different stages of purification. (a) Urea-phosphate soluble apoVLDL. Eluate of heparin-agarose affinity chromatography (b), DEAE-cellulose chromatography (c), and preparative SDS polyacrylamide gel electrophoresis $(\mathrm{d})$.

were applied to the column which was then washed with $100 \mathrm{ml}$ of urea-phosphate buffer containing $50 \mathrm{mM} \mathrm{NaCl}$. A fraction rich in apoE was eluted with urea-phosphate buffer containing $500 \mathrm{mM} \mathrm{NaCl}$. The major contaminant at this stage was albumin. This apoE-rich fraction (Fig. 1, gel B) was dialyzed against $6 \mathrm{M}$ urea, $10 \mathrm{mM}$ Tris, $1 \mathrm{mg} / \mathrm{ml}$ DTT, pH 8.0 (Tris-urea) and applied to a $0.8 \times 20$-cm DEAE-cellulose column. Elution was then carried out with a 200 -ml linear gradient of $\mathrm{NaCl}(10-50 \mathrm{mM})$ in Tris-urea. Fractions containing apoE with minimal contamination, as assessed by sodium dodecyl sulfate (SDS) polyacrylamide gel electrophoresis (Fig. 1, gel C), were pooled, concentrated by dialysis against polyethylene glycol $(20,000 \mathrm{~mol} \mathrm{wt})$, and then dialyzed against a solution of $0.05 \mathrm{M}$ Tris, $0.1 \% \mathrm{SDS}, 1 \mathrm{mg} / \mathrm{ml}$ DTT, pH 8.2. This material underwent preparative SDS polyacrylamide gel electrophoresis in a Buchler Poly Prep (Buchler Instruments Div., Searle Diagnostics Inc., Fort Lee, N. J.) as the final purification step. A continuous buffer system of $0.05 \mathrm{M}$ Tris, $0.1 \%$ SDS, pH 8.2, was used with an $11 \%$ polyacrylamide gel. Fractions containing a single band of protein on analytical SDS polyacrylamide gel electrophoresis were pooled (Fig. 1, gel D). Recovery of apoE, assessed by radioimmunoassay, was 93\% for the heparin affinity chromatography step, $50 \%$ for the DEAE-cellulose chromatography step, and $68 \%$ for the electrophoretic step; total recovery was $32 \%$.

\section{Preparation of antisera}

ApoE (300 $\mu \mathrm{g}$ in $1 \mathrm{ml}$ ) was emulsified with $1 \mathrm{ml}$ of Freund's complete adjuvant and injected intradermally into each of five male New Zealand white rabbits at 2-3-wk intervals. The rabbits were bled $7-10 \mathrm{~d}$ after the second and third injections.

\section{Preparation of ${ }^{125} \mathrm{I}-\mathrm{A}$ poE}

ApoE was iodinated by the procedure of Greenwood et al. (20) as modified by Fainaru et al. (21). ApoE was dialyzed against a solution of $0.1 \mathrm{M} \mathrm{Na}$ phosphate, $0.1 \mathrm{M} \mathrm{Na}$ decyl sulfate, $\mathrm{pH}$ 7.4. To $10 \mu \mathrm{l}$ of apoE solution $(0.3 \mathrm{mg} / \mathrm{ml})$ were added $20 \mu \mathrm{l}$ of $0.1 \mathrm{M}$ phosphate, $0.1 \mathrm{M}$ decyl sulfate, $\mathrm{pH} 7.4$, and $0.5 \mathrm{mCi}$ of carrier-free ${ }^{125} \mathrm{I}$. $50 \mu \mathrm{g}$ of chloramine- $\mathrm{T}$ was added, and $10 \mathrm{~s}$ later the iodination reaction was stopped by the addition of $50 \mu \mathrm{g}$ Na metabisulfite. 
Approximately $1 \mathrm{ml}$ of a solution containing $0.05 \mathrm{M}$ Na phosphate, $0.1 \mathrm{M} \mathrm{NaCl}, 0.02 \% \mathrm{Na}$ azide (PBS), $1 \%$ bovine serum albumin (BSA), $0.1 \mathrm{M}$ Na decyl sulfate, pH 7.4 (PBS/BSA/0.1 $M$ decyl sulfate) was added and free iodine was removed by chromatography on a $0.8 \times 18$-cm column of Sephadex G-25 equilibrated and eluted with PBS/BSA/0.1 M decyl sulfate. Efficiency of labeling was 20-45\%, giving not $>1$ mol of iodine bound per mole of apoE.

\section{Radioimmunoassay of ApoE}

The assay was carried out in $10 \times 75-\mathrm{mm}$ glass test tubes by a modification of the procedure of Fainaru et al. (21). The contents of each tube had final concentrations of $50 \mathrm{mM} \mathrm{Na}$ phosphate, $100 \mathrm{mM} \mathrm{NaCl}, 5 \mathrm{mM}$ decyl sulfate, $0.02 \% \mathrm{Na}$ azide, pH 7.4, and a total volume of $0.5 \mathrm{ml}$. Each tube included (a) $0.055 \mu \mathrm{l}$ antiserum, (b) $0.2 \mu \mathrm{l}$ nonimmune rabbit serum, (c) $50 \mu$ l of sample which had been preincubated overnight in PBS/BSA/50 mM Na decyl sulfate, and $(d) 15,000 \mathrm{cpm}{ }^{125} \mathrm{I}$ apoE. The assay tubes were incubated for $2 \mathrm{~d}$ at $4^{\circ} \mathrm{C}$, and then $100 \mu \mathrm{l}$ of a $1 / 100$ dilution of goat anti-rabbit serum in PBS/BSA was added to each tube. Following an additional overnight incubation, the tubes were centrifuged for $100,000 \mathrm{~g}$-min and the supernates were aspirated. The precipitates were resuspended in PBS and recentrifuged. Supernates were again aspirated. Radioactivity in the precipitates was measured in a Packard model 3,002 gamma counter (Packard Instrument Co., Inc., Downers Grove, Ill.).

All samples were assayed in duplicate. Each assay contained as controls: $(a)$ tubes to which no unlabeled apoE had been added, $(b)$ tubes to which an excess of antiserum $(1 \mu \mathrm{l})$ had been added (these were harvested with $12.5 \mu \mathrm{l}$ of undiluted goat anti-rabbit serum), and (c) tubes to which no anti-apoE antiserum had been added.

Because it had been suggested that the immunoreactivity of highly purified apolipoproteins might be less stable than that of apolipoproteins in unprocessed plasma (22), standard curves were prepared from a calibrated plasma pool which was stored in ampules at $-80^{\circ} \mathrm{C}$. Each assay contained three prepared standard curves, each pipetted in duplicate and each containing 15 different concentrations of apoE. The plasma secondary standard which was routinely used to prepare these curves was calibrated by radioimmunoassay against three different preparations of highly purified apoE. The third of these pools of purified apoE was purified and used to assay the secondary standard 12 mo after the first. The protein content of the primary purified apoE standards was determined by the method of Lowry et al. (23) using gravimetric standards of BSA. The values of apoE concentration in the plasma secondary standard which were obtained by assay against the three individual pools of purified apoE were: $30.8,29.7$, and $30.7 \mu \mathrm{g} / \mathrm{ml}$. The mean of these values, $30.4 \mu \mathrm{g} / \mathrm{ml}$, was taken as the true concentration of apoE in the secondary standard.

The radioimmunoassay results were calculated by a modification of the procedure of Rodbard and Hutt (24) using a previously described nonlinear estimation procedure for determination of the parameters of the standard curve (25). The residual standard deviation of the points of the standard curves about the fitted expression ranged from 1.8 to $3.5 \%$.

\section{Fractionation of plasma lipoproteins}

Agarose column chromatography. Whole plasma (1-2 ml) was applied to a $1 \times 120$-cm column of $6 \%$ agarose (BioGel A5M, BioRad Laboratories, Richmond, Calif.) and lipoproteins were eluted with a solution of $0.2 \mathrm{M} \mathrm{NaCl}, 1 \mathrm{mM}$ EDTA, $2 \mathrm{mM} \mathrm{Na}$ phosphate, $0.02 \% \mathrm{Na}$ azide, $\mathrm{pH} \mathrm{7.4}$. Plasma was never more than $10 \mathrm{~d}$ old at the time of chromatography.
Preparative ultracentrifugation. Aliquots of each sample of plasma were adjusted to the six following densities: 1.006, $1.019,1.050,1.063,1.125$, and $1.21 \mathrm{~g} / \mathrm{ml}(16)$. Each aliquot underwent a single ultracentrifugation at $40,000 \mathrm{rpm}$ at $4^{\circ} \mathrm{C}$ in a Beckman 40.3 rotor. The three least dense fractions were centrifuged for $24 \mathrm{~h}$; the others were centrifuged for $48 \mathrm{~h}$. Top and bottom fractions obtained by tube slicing were assayed for apoE. Recovery of apoE averaged $89 \%$. The distribution of apoE in density ranges was determined by difference. This procedure, which subjected each aliquot of plasma to only a single ultracentrifugation, minimized artifacts which ultracentrifugation may impart to the determination of the lipoprotein distribution of apoE.

\section{Other analytical techniques}

Analytical SDS polyacrylamide gel electrophoresis was performed in gels containing $6 \%$ acrylamide and $0.5 \%$ methylene bisacrylamide using a previously described continuous buffer system (17). Samples were incubated with $1 \%$ mercaptoethanol before electrophoresis. The gels were stained by the method of Weber and Osborne (26).

Protein was measured by the method of Lowry et al. (23) using BSA as standard. Cholesterol in extracts of agarose column fractions was measured by the procedure of Chiamori and Henry (27). Cholesterol and triglyceride concentration in plasma were determined using Technicon AA1 methodology (Technicon Instruments Corp., Tarrytown, N. Y.) (28). HDL cholesterol in plasma samples was measured by the procedure specified for the Lipid Research Clinics (29). Double immunodiffusion was carried out using gels prepared from $1 \%$ agarose in $50 \mathrm{mM} \mathrm{Na}$ barbital, $100 \mathrm{mM} \mathrm{NaCl}, 0.01 \% \mathrm{Na}$ azide, $\mathrm{pH}$ 8.0.

Isoelectric focusing was carried out as described by Warnick et al. (30). Gels were fixed for $48 \mathrm{~h}$ in a solution of $5 \%$ sulfosalicylic acid, 5\% trichloroacetic acid and then stained with Coomassie blue G-250 $(0.4 \mathrm{mg} / \mathrm{ml})$ in a solution of perchloric acid $(35 \mathrm{mg} / \mathrm{ml})$. After $24 \mathrm{~h}$ of staining, the gels were transferred to tubes containing a solution of 5\% methanol, $7.5 \%$ acetic acid which intensified the protein staining. The gels were scanned at $560 \mathrm{~nm}$, and peak areas were measured by planimetry.

Amino acid analyses were performed on a Beckman model $121 \mathrm{MB}$ amino acid analyzer using a single column program provided by Beckman Instruments (31). Protein samples underwent $24 \mathrm{~h}$ hydrolyses in $6 \mathrm{~N} \mathrm{HCl}$ at $110^{\circ} \mathrm{C}$ before amino acid analysis.

\section{RESULTS}

Purified ApoE. The purified apoE was identified by its mobility in SDS polyacrylamide gel electrophoresis and by an amino acid composition (Table I) similar to that reported by several other investigators (1-4). The final preparation appeared to be completely pure as assessed by SDS polyacrylamide gel electrophoresis. The purified apoprotein did not react in double immunodiffusion with antisera to apoA-I, apoA-II, apoB, apoC-II, or apoC-III. Furthermore, it produced monospecific antisera when injected into rabbits.

Anti-ApoE antisera. Monospecific antisera against apoE were obtained from each of five rabbits. The antisera produced a single line of identity in immunodiffusion against apoE and the apoproteins of VLDL. 
TABLE I

Amino Acid Composition of ApoE

\begin{tabular}{lr}
\hline \multicolumn{1}{c}{ Amino acid } & $\begin{array}{c}\text { Residues/100 residues } \\
\text { analyzed }\end{array}$ \\
\hline Aspartic acid & 4.63 \\
Threonine & 3.94 \\
Serine & 4.80 \\
Glutamic acid & 23.10 \\
Proline & 2.87 \\
Glycine & 6.30 \\
Alanine & 11.59 \\
Valine & 7.74 \\
Methionine & 2.10 \\
Isoleucine & 0.88 \\
Leucine & 12.96 \\
Tyrosine & 1.29 \\
Phenylalanine & 1.10 \\
Histidine & 0.81 \\
Lysine & 4.28 \\
Arginine & 11.54 \\
\hline
\end{tabular}

The values listed are the means of four determinations.

The antiserum with the highest titer and affinity was selected for use in the radioimmunoassay. None of the antisera reacted in double immunodiffusion against apoA-I, apoA-II, apoB, apoC-II, apoC-III, or human serum albumin. Further evidence of the specificity of the antiserum used in the radioimmunoassay was obtained in the studies of cross-reactivity in the radioimmunoassay.

Radioimmunoassay for ApoE. The anti-apoE antiserum was capable of precipitating $60-65 \%$ of freshly iodinated apoE. Immunoprecipitability of ${ }^{125} \mathrm{I}$-apoE diminished to about $45 \%$ at the end of $6 \mathrm{wk}$, when a new iodination was generally performed.

Plasma and purified apoE produced identical displacement curves as demonstrated in Fig. 2. Although perceptible displacement occurred with as little as 0.5 ng of apoE, the effective working range of the assay was $0.8-12 \mathrm{ng}$.

Coefficients of variation for the radioimmunoassay were determined from six separate runs. In each run, measurement of the apoE content of a sample of plasma was replicated five times. For each run a coefficient of variation was calculated from the five values obtained. The overall within-assay coefficient of variation was taken as the mean of the five coefficients of variation for the different runs and was $9 \%$. To determine the coefficient of variation for systematic between-assay variability, the mean of the five replicates was calculated for each run and the coefficient of variation of the six resulting means was used; this was $3 \%$.

The assay was validated by measurement of apoE concentration in a freshly prepared sample of VLDL in which the apoE content was also measured by scan-

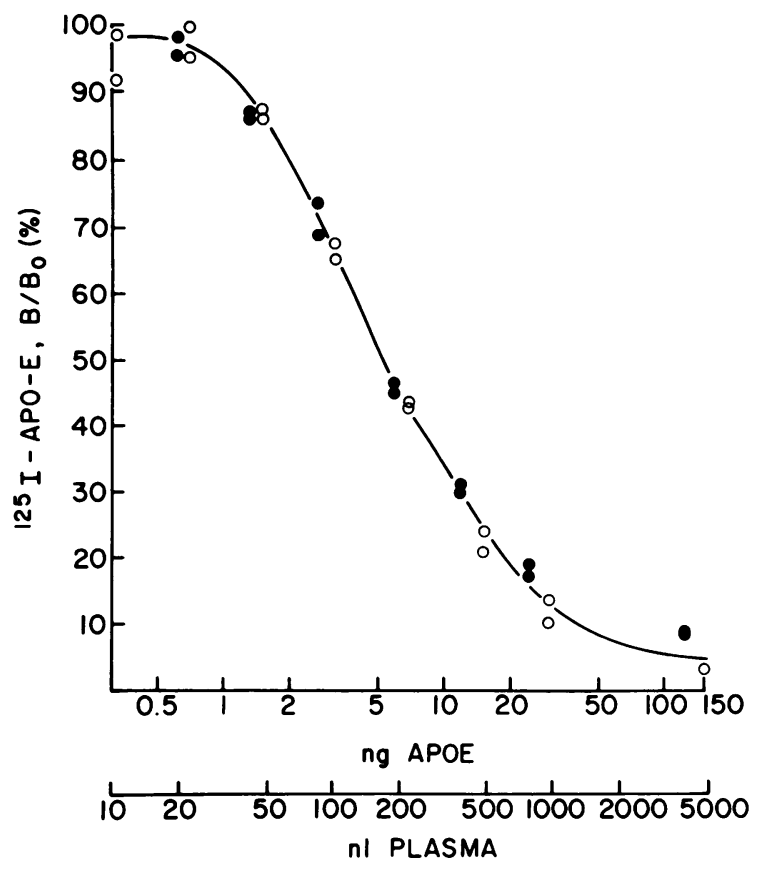

Figure 2 Radioimmunoassay of apoE. The plot shows the inhibition of antibody-binding of ${ }^{125} \mathrm{I}$-apoE by purified apoE $(O)$ or human plasma $(O) . \mathrm{B} / \mathrm{B}_{0}$, precipitated radioactivity as a percentage of that which is precipitated in the absence of unlabeled apoE.

ning stained SDS gels. For this experiment, duplicate aliquots of six different masses of purified apoE $(0.3-3$ $\mu \mathrm{g})$ were electrophoresed and stained concurrently with measured volumes of VLDL. The stained gels were scanned at $550 \mathrm{~nm}$ and peak areas were measured by planimetry. A standard curve constructed from the masses and peak areas of purified apoE had a linear correlation coefficient of 0.996 . The concentration of apoE in VLDL was calculated from the area of the apoE peaks in gels into which VLDL was electrophoresed. The result obtained by radioimmunoassay using a purified apoE standard was $104 \%$ of that obtained by quantitative gel densitometry.

Delipidated and undelipidated plasma and lipoprotein fractions gave identical displacement curves. Repeated freezing and thawing of plasma samples (up to six times) did not alter their behavior in the radioimmunoassay. Furthermore, the conditions of storage (frozen or refrigerated, undiluted or diluted in a solution with $50 \mathrm{mM} \mathrm{Na}$ decyl sulfate) did not affect the assay of plasma. Storage of frozen plasma for up to 15 mo or refrigerated plasma for up to 6 mo did not affect the immunoassayable apoE.

Cross-reactivity from apoA-I, apoA-II, apoB, apoC-I, apoC-II, apoC-III, and human albumin was less than $0.1 \%$, i.e., purified preparations of these apoproteins had $<0.1 \%$ the potency of apoE in inhibiting the binding 
of ${ }^{125} \mathrm{I}$-apoE to antibody. Such small amounts of measured cross-reactivity could have been due to trace amounts of apoE present in the preparations of the other apoproteins, amounts so small as to be detectable only by radioimmunoassay.

Plasma from patients with different ratios of the isoforms of apoE gave parallel displacement curves. Moreover, purified preparations of apoE from individual patients enriched or depleted in different isoforms had identical immunoreactivity. Thus, this radioimmunoassay does not appear to distinguish among the isoforms, and results obtained validly represent total apoE concentrations.

ApoE concentration in plasma. The plasma apoE levels found in normal and hyperlipidemic subjects are summarized in Table II. The mean apoE concentration of the normal subjects was $36 \pm 13 \mu \mathrm{g} / \mathrm{ml} \mathrm{SD}$. The mean in males $(n=17)$ did not differ from that in females. In the 26 normals, apoE concentration did not correlate with HDL cholesterol concentration. It did, however, correlate significantly with both plasma cholesterol $(r=0.54, P<0.01)$ and triglyceride $(r=0.41, P<0.02)$ concentrations. As might be predicted from this result among the normals, higher mean apoE levels were found in all groups of hyperlipidemic patients. For the purposes of this analysis, our patients were segregated by the arbitrary cutoffs of $275 \mathrm{mg} / \mathrm{dl}$ for cholesterol and $250 \mathrm{mg} / \mathrm{dl}$ for triglycerides. The six patients with triglyceride concentrations $>1,000 \mathrm{mg} / \mathrm{dl}$ had fasting chylomicronemia and generally had extremely high apoE levels. Since it appeared that they might be qualitatively different from the others, they were treated as a separate group for data analysis in Table II. Similarly, the one patient with type III hyperlipoproteinemia (VLDL cholesterol/triglyceride ratio 0.47 , apo $E_{\text {III }}$ deficiency on isoelectric focusing) was excluded from other groups and is listed separately. His plasma apoE concentration of $664 \mu \mathrm{g} / \mathrm{ml}$ is the highest in this series.

Although the hypertriglyceridemic patients had the highest mean apoE levels of all of the nonchylomicronemic groups, and those with hypertriglyceridemia alone had a mean apoE level nearly twice that of the normals, there were several patients with hypertriglyceridemia alone or with hypercholesterolemia alone who had apoE levels below the mean value for the group of 26 normals. The patient with the highest plasma cholesterol in this series $(469 \mathrm{mg} / \mathrm{dl}) \mathrm{had}$ an apoE level of $53 \mu \mathrm{g} / \mathrm{ml}$, well below the mean for patients with hypercholesterolemia alone. Thus, a very high plasma cholesterol or triglyceride concentration does not guarantee an elevated apoE concentration. The mean apoE level of those with familial hypercholesterolemia $(n=7$, apoE $=56 \pm 22 \mu \mathrm{g} / \mathrm{ml} \mathrm{SD})$ did not differ significantly from that of other patients with hypercholesterolemia alone $(n=22$, apoE $=63 \pm 15 \mu \mathrm{g} / \mathrm{ml} \mathrm{SD})$ $(P=0.16)$.

Since in all of the nonchylomicronemic groups there seemed to be similar quantitative relationships between plasma lipid levels and apoE levels, their data were pooled for further analysis with data from the normals and from other patients whose lipid levels fell below the arbitrary cutoffs. A total population of 108 nonchylomicronemic persons was studied. Fig. 3 shows the relationships observed between apoE concentration and fasting plasma cholesterol or triglyceride concentration in this group. The simple correlations of apoE with plasma cholesterol $(r=0.35, P=0.0001)$ and triglyceride $(r=0.65, P<0.0001)$ were highly significant. However, the known correlation between plasma cholesterol and triglyceride levels in populations might make such a simple analysis misleading. A multiple regression analysis was therefore performed. This analysis yielded the following equation: $\mathrm{ApoE}(\mu \mathrm{g} / \mathrm{ml})$ $=0.15 \mathrm{Chol}+0.088 \mathrm{Tg}+2.05$. In this equation, Chol and $\mathrm{Tg}$ are the plasma cholesterol and triglyceride concentration in milligrams per deciliters. The coefficients for cholesterol and triglycerides are both highly significantly different from zero. If cholesterol and triglyceride concentrations are expressed in units of

TABLE II

Fasting Plasma ApoE Concentration in Normal and Hyperlipidemic Subjects

\begin{tabular}{|c|c|c|c|c|}
\hline & Number & ApoE* & Cholesterol* & Triglycerides \\
\hline & & $\mu g / m l$ & $m g / d l$ & $m g / d l$ \\
\hline Normals & 26 & $36 \pm 13$ & $187 \pm 34$ & $101 \pm 55$ \\
\hline Cholesterol > 275 & & & & \\
\hline Triglycerides $\leqslant 250$ & 29 & $63 \pm 18$ & $316 \pm 40$ & $129 \pm 51$ \\
\hline Cholesterol $\leqslant 275$ & & & & \\
\hline $1,000>$ Triglycerides $>250$ & 26 & $81 \pm 31$ & $233 \pm 29$ & $466 \pm 184$ \\
\hline Cholesterol >275 & & & & \\
\hline $1,000>$ Triglycerides $>250$ & 6 & $72 \pm 19$ & $313 \pm 32$ & $425 \pm 257$ \\
\hline $\begin{array}{l}\text { Triglycerides } \geqslant 1,000 \\
\quad \text { (with fasting chylomicrons) }\end{array}$ & 6 & $244 \pm 217$ & $315 \pm 129$ & $3,548 \pm 2,746$ \\
\hline Type III & 1 & $664-$ & $394-$ & $516-$ \\
\hline
\end{tabular}

The values listed are mean $\pm \mathrm{SD}$. 


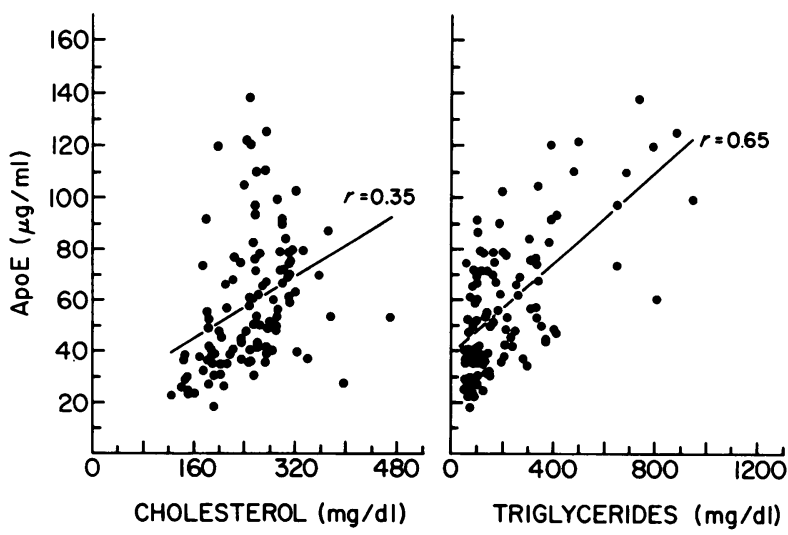

Figure 3 Relationship between plasma apoE concentration and plasma cholesterol (left panel) and triglyceride (right panel) concentrations in 108 normal volunteers and nonchylomicronemic hyperlipidemic patients.

their standard deviation in this population, the equation reads as follows: $\mathrm{ApoE}(\mu \mathrm{g} / \mathrm{ml})=9.16 \mathrm{Chol}+16.9$ $\mathrm{Tg}+2.05$. That the coefficient for triglycerides is greater than that for cholesterol when the independent variables are expressed in terms of their standard deviations indicates that in this population the triglyceride concentration is the more important predictor of apoE level. This relationship has a multiple correlation coefficient of 0.73 , indicating that in this population $53 \%$ (the square of the multiple correlation coefficient) of the variability in apoE concentration is described by variation in total plasma cholesterol and triglyceride levels.

Distribution of apoE among lipoproteins. The distribution of apoE among the plasma lipoproteins was investigated in normal subjects and in hyperlipidemic patients both by agarose column chromatography (Figs. 4 and 5) and by preparative ultracentrifugation (Table III).

Whole plasma passed over a 6\% agarose column produced one of two distinct patterns. Plasma from normal volunteers (nine samples chromatographed) and from hypercholesterolemic patients (three samples chromatographed) produced a pattern similar to that shown in Fig. 4. In this pattern, apoE eluted in two peaks. The first peak of apoE immunoreactivity coincided with the elution volume for VLDL. A second and considerably larger peak of apoE immunoreactivity overlapped the ascending limb of the peak of HDL cholesterol, indicating that the lipoproteins containing apo $E$ in this peak have a somewhat larger average Stokes radius than typical HDL, but one that is distinctly smaller than that of LDL. This class of apoEcontaining lipoproteins is here referred to as apoE-HDL. Integration of the areas under the curves indicated that in normals $64 \pm 16 \% \mathrm{SD}$ of apoE immunoreactivity eluted with the second peak (apoE-HDL) and that in hypercholesterolemics $74 \pm 12 \%$ SD of apoE immunoreactivity eluted with apoE-HDL.

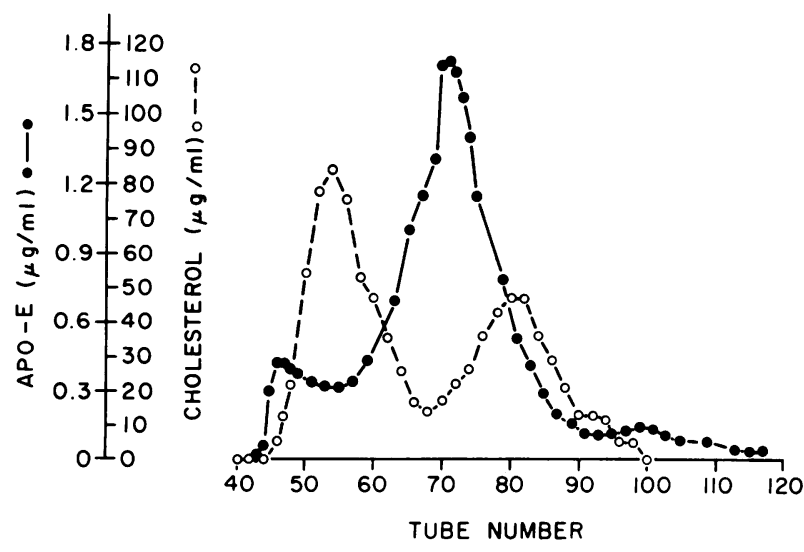

Figure 4 Agarose column chromatography of normal plasma. Plasma was applied to a $1 \times 120-\mathrm{cm}$ column of $6 \%$ agarose and was eluted with a solution of $0.2 \mathrm{M} \mathrm{NaCl}, 1 \mathrm{mM}$ EDTA, $2 \mathrm{mM}$ Na phosphate, $0.02 \% \mathrm{Na}$ azide, pH 7.4. ApoE and cholesterol concentrations were measured in the eluate. The first peak of cholesterol represents LDL, the second peak represents HDL. VLDL and chylomicrons elute from the column in fractions $45-50$.

Plasma from hypertriglyceridemic patients (five samples chromatographed) produced a second and very different pattern (Fig. 5). Nearly all apoE immunoreactivity eluted with the triglyceride-rich lipoproteins.

The reproducibility of the column separation was tested by analyzing eluates produced from five aliquots of the same pool of plasma. The five aliquots were chromatographed sequentially; the first aliquot was applied to the column the day the plasma was obtained from a normal donor; the fifth aliquot was applied $18 \mathrm{~d}$ later. The amount of apoE appearing in the first peak as a percent of the total appearing in the

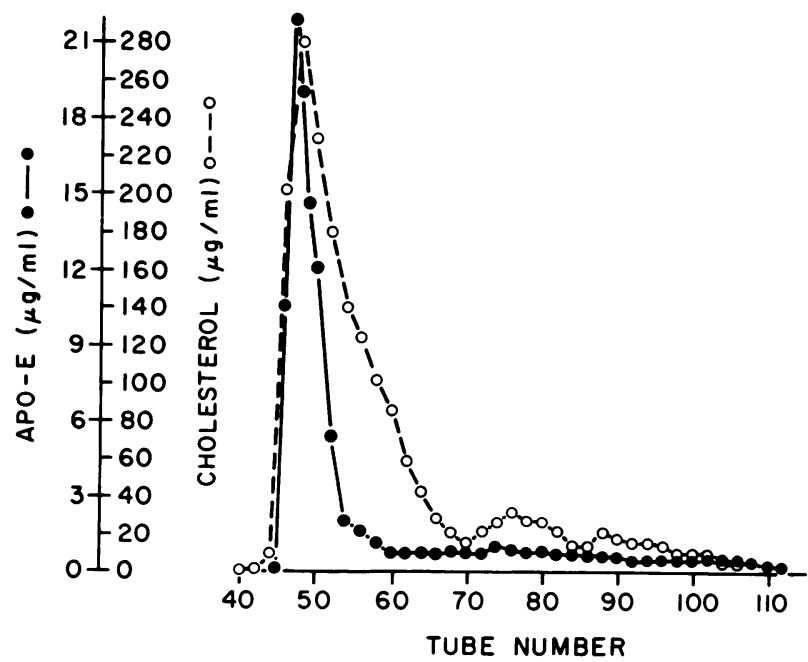

Figure 5 Agarose column chromatography of plasma from a hypertriglyceridemic patient. Chromatography was performed as described in the legend to Fig. 4. 
TABLE III

\begin{tabular}{|c|c|c|c|c|c|c|c|c|c|c|c|c|}
\hline \multirow[b]{2}{*}{ Subject } & \multirow[b]{2}{*}{$\begin{array}{l}\text { Choles- } \\
\text { terol }\end{array}$} & \multirow[b]{2}{*}{$\begin{array}{l}\text { Triglycer- } \\
\text { ides }\end{array}$} & \multirow[b]{2}{*}{ ApoE } & \multicolumn{6}{|c|}{$\begin{array}{l}\text { Percent distribution of lipoprotein-associated } \\
\text { ApoE in density ranges }\end{array}$} & \multirow{2}{*}{$\begin{array}{c}\text { Percent } \\
\text { of plasma } \\
\text { ApoE } \\
d>1.21\end{array}$} & \multicolumn{2}{|c|}{$\begin{array}{l}\text { Agarose column } \\
\text { chromatography }\end{array}$} \\
\hline & & & & $d<1.006$ & $\begin{array}{c}1.006- \\
1.019\end{array}$ & $\begin{array}{l}1.019- \\
1.050\end{array}$ & $\begin{array}{c}1.050- \\
1.063\end{array}$ & $\begin{array}{c}1.063- \\
1.125\end{array}$ & $\begin{array}{c}1.125- \\
1.21\end{array}$ & & Peak 1 & Peak 2 \\
\hline & $m g / d l$ & $m g / d l$ & $\mu g / m l$ & & & $g / m$ & & & & $g / m l$ & $\% *$ & $\% *$ \\
\hline \multicolumn{13}{|c|}{ Normals } \\
\hline 1 & 212 & 125 & 35 & 60.3 & 7.9 & 11.1 & 0.0 & 9.5 & 11.1 & 37.0 & & \\
\hline 2 & 151 & 88 & 24 & 24.4 & 1.3 & 17.9 & 41.0 & 1.3 & 14.1 & 22.0 & & \\
\hline 3 & 144 & 55 & 37 & 10.4 & 1.5 & 11.6 & 9.6 & 43.9 & 23.0 & 28.2 & 14.5 & 85.5 \\
\hline 4 & 169 & 88 & 38 & 42.3 & 0.3 & 9.9 & 2.8 & 31.8 & 12.9 & 27.1 & & \\
\hline 5 & 234 & 57 & 75 & 15.0 & 4.1 & 17.2 & 0.0 & 37.2 & 26.5 & 31.6 & & \\
\hline 6 & 209 & 129 & 66 & 8.3 & 3.3 & 14.0 & 4.7 & 59.7 & 9.9 & 30.2 & & \\
\hline 7 & 154 & 76 & 32 & 15.1 & 11.2 & 14.5 & 7.7 & 43.8 & 7.8 & 23.2 & 38.8 & 41.2 \\
\hline 8 & 181 & 112 & 41 & 33.9 & 0.0 & 8.3 & 6.7 & 29.8 & 21.3 & 16.5 & 42.7 & 57.3 \\
\hline 9 & 159 & 61 & 37 & 23.9 & 12.5 & 19.8 & 3.0 & 38.9 & 1.9 & 29.8 & 39.9 & 40.1 \\
\hline Mean & 179 & 88 & 43 & 26.0 & 4.7 & 13.8 & 8.4 & 32.9 & 14.3 & 27.3 & & \\
\hline SD & 32 & 29 & 17 & 17.0 & 4.7 & 3.9 & 12.7 & 17.9 & 7.9 & 6.0 & & \\
\hline \multicolumn{13}{|c|}{ Hypercholesterolemics } \\
\hline 10 & 372 & 103 & 87 & 24.4 & 12.2 & 12.4 & 0.0 & 46.5 & 4.5 & 42.7 & & \\
\hline 11 & 415 & 101 & 53 & 14.0 & 1.8 & 42.2 & 0.0 & 29.9 & 12.1 & 31.3 & 35.0 & 65.0 \\
\hline 12 & 296 & 165 & 79 & 21.5 & 12.4 & 23.2 & 9.1 & 29.5 & 4.4 & 26.4 & & \\
\hline $13 \ddagger$ & 329 & 198 & 103 & 57.0 & 11.8 & 28.0 & 0.0 & 3.8 & 0.0 & 46.7 & & \\
\hline 14 & 299 & 199 & 66 & 20.9 & 5.1 & 30.8 & 0.0 & 40.6 & 2.5 & 26.8 & & \\
\hline 15 & 332 & 113 & 80 & 15.3 & 0.0 & 29.1 & 1.8 & 46.6 & 7.0 & 55.0 & 17.2 & 82.8 \\
\hline 16 & 313 & 88 & 61 & 24.5 & 8.5 & 19.4 & 18.5 & 29.1 & 0.0 & 54.3 & & \\
\hline 17 & 253 & 209 & 57 & 34.0 & 21.1 & 7.1 & 3.5 & 19.6 & 14.7 & 17.1 & & \\
\hline 18 & 263 & 166 & 31 & 33.6 & 23.3 & 8.8 & 0.0 & 28.0 & 6.9 & 25.8 & & \\
\hline Mean & 319 & 149 & 69 & 27.2 & 10.7 & 22.3 & 3.7 & 30.4 & 5.8 & 36.2 & & \\
\hline SD & 51 & 48 & 21 & 13.1 & 7.9 & 11.6 & 6.3 & 13.5 & 5.0 & 13.7 & & \\
\hline \multicolumn{13}{|c|}{ Hypertriglyceridemics } \\
\hline 19 & 177 & 761 & 73 & 83.9 & 6.6 & 0.0 & 0.0 & 5.5 & 4.0 & 33.7 & & \\
\hline 20 & 211 & 550 & 109 & 67.5 & 13.3 & 5.4 & 0.0 & 10.3 & 3.4 & 12.3 & & \\
\hline 21 & 216 & 344 & 89 & 70.0 & 9.4 & 5.2 & 0.0 & 13.0 & 2.5 & 17.3 & & \\
\hline 22 & 185 & 417 & 79 & 66.6 & 14.2 & 0.0 & 0.0 & 11.7 & 7.5 & 32.2 & & \\
\hline 23 & 263 & 301 & 80 & 93.4 & 0.0 & 0.0 & 0.0 & 0.0 & 6.6 & 56.7 & & \\
\hline 24 & 219 & 295 & 53 & 88.9 & 0.0 & 0.0 & 3.2 & 7.9 & 0.0 & 45.7 & & \\
\hline 25 & 174 & 355 & 86 & 92.7 & 1.0 & 0.0 & 0.0 & 0.0 & 6.3 & 57.1 & & \\
\hline 26 & 138 & 377 & 37 & 79.6 & 3.4 & 4.5 & 5.7 & 6.8 & 0.0 & 19.0 & & \\
\hline 27 & 203 & 2226 & 125 & 95.6 & 0.0 & 0.6 & 0.3 & 1.6 & 1.9 & 6.6 & 100.0 & 0.0 \\
\hline Mean & 198 & 625 & 81 & 82.1 & 5.3 & 1.7 & 1.0 & 6.3 & 3.6 & 31.2 & & \\
\hline SD & 35 & 618 & 26 & 11.6 & 5.8 & 2.5 & 2.0 & 4.9 & 2.8 & 18.9 & & \\
\hline
\end{tabular}

Aliquots of plasma were adjusted to the following densities: 1.006, 1.019, 1.050, 1.063, 1.125, and 1.21 g/ml. Each aliquot underwent a single ultracentrifugation. ApoE distribution in density ranges was determined by difference and is expressed as percent of that apoE which is in $d<1.21 \mathrm{~g} / \mathrm{ml}$.

* Peak 1 represents percent of eluted apoE in triglyceride-rich lipoproteins. Peak 2 represents percent of apoE in HDL.

† Diabetic.

first and second peaks was: $51.1 \pm 1.2 \%$. There was no trend in this statistic from the first sample $(50.0 \%)$ to the fifth $(50.8 \%)$. However, as the samples aged, apoE immunoreactivity did appear in nonlipoprotein fractions. None was apparent in the initial chromatography, but this amounted to $4 \%$ of total eluted immunoreac- tivity in the sample chromatographed when 1 wk old and $7.1 \%$ of eluted immunoreactivity in the sample chromatographed when $18 \mathrm{~d}$ old. Since the ratio of peak 1 (VLDL) to peak 2 (apoE-HDL) did not change between the first and last samples, it is apparent that the nonlipoprotein apoE, which appeared over time, 
was derived from VLDL and HDL in approximately equal amounts. Recovery of apoE immunoreactivity in these five analyses averaged $87.3 \pm 3.0 \%$.

In the agarose column studies nearly all apoE immunoreactivity was associated with lipoproteins. In no case did $>8 \%$ of apoE immunoreactivity elute after apoE-HDL.

Since preparative ultracentrifugation permits fractionation of a larger number of samples and gives results more amenable to quantitative analysis than column chromatography, we also fractionated normal and hyperlipidemic plasma by ultracentrifugation (Table III). The same two patterns of apoE distribution found in the column chromatographic studies were also evident with preparative ultracentrifugation. In the first pattern seen in normal volunteers and hypercholesterolemic patients, apoE was found concentrated in two distinct density ranges, the VLDL-chylomicron density range and a higher density range. In the second pattern, seen in hypertriglyceridemic subjects, nearly all apoE was found associated with triglyceride-rich lipoproteins.

In the first pattern (normal and hypercholesterolemic subjects), apoE in the higher density range corresponds to the second peak of apoE immunoreactivity in the agarose column studies (Fig. 4) and accounted for a majority of lipoprotein-associated apoE of these subjects. The portion of lipoprotein-associated apoE found in the $d<1.006 \mathrm{~g} / \mathrm{ml}$ fraction of plasma was similar in normal subjects and in hypercholesterolemic patients. In these studies of the lipoprotein density distribution of apoE, the apoE-HDL was most heavily concentrated in the $\mathrm{HDL}_{2}$ density range (1.063-1.125 $\mathrm{g} / \mathrm{ml})$. This finding is in accordance with the results of the column chromatographic studies, where apoE-HDL coincided with the ascending limb of the peak of HDL cholesterol.

The large standard deviations in Table III indicate a large amount of variability between individuals in apoE distribution according to lipoprotein density, especially among the normal subjects and the hypercholesterolemic patients. This variability particularly reflects the fact that some of the normal and hypercholesterolemic subjects did not have a major portion of their apoE in the $\mathrm{HDL}_{2}$ density range. For example, one of the normals (subject 1 ) had $60 \%$ of his lipoprotein-associated apoE in the VLDL density range and he had very little elsewhere; another normal (subject 2) had most of his apoE-HDL in the very narrow density range $1.050-1.063 \mathrm{~g} / \mathrm{ml}$. In several of the hypercholesterolemic patients, a large amount of lipoprotein-associated apoE was in the major LDL density range $(1.019-1.050 \mathrm{~g} / \mathrm{ml})$ and on average more apoEHDL was in this density range in hypercholesterolemics than in normals $(P=0.039)$. This suggested that in hypercholesterolemic patients apoE-HDL is on average more buoyant than in normals. Indeed, hyper- cholesterolemic patients have smaller portions of their plasma apoE in the $\mathrm{HDL}_{3}$ density range than do normals $(P=0.005)$. Nevertheless, the altered density distribution of apoE-HDL among the hypercholesterolemic patients was not reflected in greatly altered elution volumes in agarose column chromatography. Thus, in every instance where agarose gel filtration was carried out (as well as ultracentrifugal separation), two peaks of eluted apoE were seen, with the second peak located after the LDL peak (as in Fig. 4), regardless of variability in the ultracentrifugal density distribution. It is apparent that the second peak of apoE immunoreactivity varies less in molecular size than in hydrated density, among normal and hypercholesterolemic subjects. However, it does appear that persons with the greatest amount of apoE in the 1.019-1.050 $\mathrm{g} / \mathrm{ml}$ density range do have a slightly larger mean particle size for their apoE-HDL. Thus, for subjects 11 and 15 in Table III, the elution volume for the peak of apoE-HDL was 102 and $104 \mathrm{ml}$, respectively. This compares to a mean elution volume of $108 \pm 3.7 \mathrm{ml}$ SD for plasma from the 12 different normal and hypercholesterolemic subjects examined with this column. Of these 12 samples, those of subjects 11 and $15 \mathrm{had}$ apoE-HDL eluting the earliest.

As indicated above, the density distribution of apoE in the plasma of hypertriglyceridemic patients was very different from that seen in normal volunteers and hypercholesterolemic patients. Nearly all apoE was associated with the triglyceride-rich lipoproteins.

In the ultracentrifugation studies, a mean of $32 \%$ of immunoreactive apoE was found in the $d>1.21$ $\mathrm{g} / \mathrm{ml}$ fraction of plasma. The portion of plasma apoE found in this fraction was similar in normal $(27.3 \pm 6.0 \%$ $\mathrm{SD})$, hypercholesterolemic $(36.2 \pm 13.7 \% \mathrm{SD})$, and hypertriglyceridemic $(31.2 \pm 18.9 \% \mathrm{SD})$ subjects. Since in the agarose column chromatographic studies nearly all apoE was associated with lipoproteins, it is likely that the large amount in the $d>1.21 \mathrm{~g} / \mathrm{ml}$ fraction represents an artifact produced by ultracentrifugation.

In a single normal subject, apoE was isolated by heparin-agarose chromatography both from VLDL and from the $1.019-1.21 \mathrm{~g} / \mathrm{ml}$ fraction of plasma. The distribution of isoforms of apoE was then examined by isoelectric focusing and was found to be similar in these two fractions $\left(\mathrm{apoE}_{\mathrm{I}} / \mathrm{apoE}_{\mathrm{II}} / \mathrm{apoE}_{\mathrm{III}}=1.00 / 1.05 / 2.00\right.$ in VLDL and 1.00/0.98/1.75 in the $1.019-1.21 \mathrm{~g} / \mathrm{ml}$ density range).

\section{DISCUSSION}

This report describes the development and first applications of a sensitive and specific double antibody radioimmunoassay for human apoE. There have been three previous reports of immunoassays for apoE. Curry et al. (12) described an electroimmunoassay, Kushwaha et al. (11) described a radial immunodiffusion assay, 
and Falko et al. (13) have recently described a radioimmunoassay. Our selection of radioimmunoassay for measurement of apoE was based in part on the much greater sensitivity (50-100-fold) of this procedure. This increased sensitivity has greatly enhanced the utility of the assay by allowing measurement of apoE concentration in column chromatographic fractions. The assay reported here, like those of Kushwaha et al. (11) and Curry et al. (12) was not affected by prior delipidation of plasma or of lipoprotein fractions; perhaps this is a result of the preincubation of samples with decyl sulfate before assay.

In assessing the plasma apoE concentration of normal and hyperlipoproteinemic subjects, we found concentrations threefold lower than did Curry et al. (12) and sevenfold lower than did Kushwaha et al. (11). Considerations of lipoprotein composition suggest that the absolute values reported here are correct. For example, in the series of Curry et al. (12), the mean plasma triglyceride concentration in a group of 48 normals was $108 \mathrm{mg} / \mathrm{dl}$. This corresponds to a VLDL cholesterol concentration of $21 \mathrm{mg} / \mathrm{dl}$ (32). Assuming that cholesterol accounts for $17 \%$ of the total mass of normal VLDL (33), this corresponds to a total VLDL mass concentration of $124 \mathrm{mg} / \mathrm{dl}$. Using Curry's figure for the fraction of total plasma apoE found in VLDL (27\%), with which we agree, and the mean plasma apoE concentration reported by Curry et al. (12) for their group of 48 normals, one calculates a VLDL apoE concentration of $2.7 \mathrm{mg} / \mathrm{dl}$. Assuming that apoE accounts for an average of $13 \%$ of normal VLDL protein (14), one calculates a VLDL protein concentration of $21 \mathrm{mg} / \mathrm{dl}$. A lipoprotein so rich in protein $(17 \%)$, however, would not have the floatation properties of VLDL. The discrepancy would be greater using the data of Kushwaha et al. (11). Similar calculations using our data yield a VLDL protein content of $8.3 \%$, a value compatible with measurements of VLDL composition and with the floatation characteristics of this class of lipoproteins. The values recently reported by Falko et al. (13) are in approximate agreement with ours.

As reported here, elevated mean apoE levels were found in all groups of hyperlipidemic patients. This is also at variance with the report of Kushwaha et al. (11), who found mean plasma apoE levels significantly above normal only in patients with type III hyperlipoproteinemia, and with the report of Curry et al. (12), who found elevated mean plasma apoE levels only in patients with type III or type V hyperlipoproteinemia. The reasons for this discrepancy are not apparent. However, our data in patients are in qualitative agreement with our data in normals since apoE levels did correlate with plasma triglyceride and cholesterol levels in the normal volunteers alone. In the entire population of 108 normal volunteers and hyperlipidemic patients (excluding one man with type III and the six patients with type $\mathrm{V}$ hyperlipoproteinemia), this correlation of plasma apoE concentration with triglyceride and cholesterol concentrations accounted for 53\% of the observed variability of apoE.

The metabolic implications of the independent relationships between apoE concentration and both plasma triglyceride and cholesterol concentration are of interest. One might speculate that elevated apoE levels in hypertriglyceridemia simply represent the presence of large amounts of VLDL or plasma chylomicrons of which apoE is a constituent. Among the hypercholesterolemics, since apoE is not particularly associated with normal LDL (the lipoprotein species in greatest excess), a more complex explanation must hold. Bersot et al. (6) have demonstrated that apoEcontaining $\mathrm{HDL}_{\mathrm{c}}$ of human origin can undergo catabolism mediated by the LDL receptor. It is thus possible that in patients with familial hypercholesterolemia, who have a deficiency of LDL receptors, retarded catabolism of apoE-containing HDL may contribute to the increased plasma levels of apoE. This, however, cannot explain the elevation of apoE levels seen in the majority of hypercholesterolemics who do not have familial hypercholesterolemia and in whom the basic metabolic lesion is not known.

Investigation of the lipoprotein distribution of apoE revealed two general patterns. In normal subjects and hypercholesterolemic patients, apoE was associated with two discrete lipoprotein fractions: $(a)$ VLDL and chylomicrons, and $(b)$ a class of lipoproteins of higher density and smaller average Stokes radius than LDL (but on average larger and less dense than average "typical" HDL), which seems analogous to the HDL observed in cholesterol-fed animals and man (7-10). The higher density fraction was generally predominant in the normals and hypercholesterolemics. In hypertriglyceridemic patients, a very different pattern was seen; nearly all plasma apoE was associated with triglyceride-rich lipoproteins.

Our observations on the lipoprotein distribution of apoE in normals are consistent with those of Curry et al. (12) and Kushwaha et al. (11). However, those investigators did not discern two discrete fractions containing apoE because they did not use column chromatographic techniques and their ultracentrifugation did not fractionate at multiple densities. In examining the lipoprotein distribution of apoE in hypertriglyceridemic patients, the results of Kushwaha et al. are different from ours; they did not find that most of lipoprotein-associated apoE was in a VL,DL-and-chylomicron fraction. It is possible that this discrepancy results from artifacts of lipoprotein structure and composition induced by the multiple-sequential ultracentrifugations to which they subjected each aliquot of plasma (34). In the studies reported here, each aliquot of plasma underwent only a single ultracentrifugation in order to avoid unnecessary artifacts. It should be noted that our conclusions about the lipoprotein distribution of 
apoE are supported by the agreement between the results obtained with two different techniques, preparative ultracentrifugation and agarose column chromatography. These studies demonstrate that all or nearly all apoE in plasma is associated with lipoproteins, although ultracentrifugation can remove large amounts to the nonlipoprotein $(d>1.21 \mathrm{~g} / \mathrm{ml})$ fraction of plasma. This observation with human apoE regarding an artifact which may be induced by ultracentrifugation is in agreement with similar studies of rat apoE performed by Fainaru et al. (21) and Mahley and Holcombe (35).

Although normal subjects and hypercholesterolemic patients all had similar patterns of distribution of apoE among lipoproteins, i.e., distribution between VLDL and apoE-HDL, there was considerable variability between individuals. The precise density at which the apoE-HDL was centered varied. This was particularly striking among some hypercholesterolemic patients in whom a large amount of apoE was found in the major LDL density range $(1.019-1.050 \mathrm{~g} / \mathrm{ml})$. Although the particles of this apoE-containing lipoprotein fraction seemed to resemble LDL in density, their size as assessed by agarose column chromatography was much smaller than that of LDL. Data from two of these hypercholesterolemic patients, however, did suggest a slightly larger mean lipoprotein particle size of apoEHDL than for normal volunteers or for hypercholesterolemic patients with the usual density profile of apoE. It seemed that in nearly every case, the apoEHDL of hypercholesterolemics was more highly lipidated than that of normals. This was indicated by the small amount of apoE found in $\mathrm{HDL}_{3}$ in the hypercholesterolemics and the larger amount found in slightly lighter fractions.

The finding that apoE-HDL accounts for a major portion of plasma apoE in normal subjects and hypercholesterolemic patients was somewhat surprising in view of the minor extent to which apoE contributes to HDL apoproteins visualized in stained SDS polyacrylamide gels. The present studies demonstrate, however, that this is because of the considerable amount of other apoproteins (primarily apoA-I and apoA-II) present in the HDL density range and that this density range does serve as a quantitatively important locus for apoE. We observed, moreover, some important differences between the distribution of apoE and that of HDL cholesterol in this density range. ApoE-HDL eluted from an agarose column distinctly in advance of the peak of HDL cholesterol, which, in turn, would tend to elute slightly in advance of the peak of apoA-I and apoA-II (36). ${ }^{2}$ Our data suggest that the lipoprotein

\footnotetext{
${ }^{2}$ Radioimmunoassay of apoA-I and apoA-II in fractions from agarose column chromatography of normal human plasma confirms that this is the case as had been suggested by the studies of Cheung and Albers (36) who used equilibrium gradient centrifugation. Blum, C. B., and L. Aron. Unpublished data.
}

particles containing apoE in this peak bear a closer resemblance to $\mathrm{HDL}_{2}$ than to $\mathrm{HDL}_{3}$ in size and density. Furthermore, since $\mathrm{HDL}_{2}$ normally accounts for a minority of the mass of HDL, and the smaller, denser $\mathrm{HDL}_{3}$ particles are the dominant species, our data indicate that apoE in higher density lipoproteins is not distributed strictly in proportion to the mass of HDL present. The difference between the size distribution of lipoproteins containing apoE in HDL and that of cholesterol in HDL suggests that apoE-HDL may represent a separate class of lipoprotein particles which contain apoE as a quantitatively major constituent. Our data do not directly address this issue.

The normal presence of apoE in two distinct classes of lipoproteins is reminiscent of the situation which holds for the C apoproteins of VLDL and HDL. The extent to which this analogy may be valid is not known. However, it is tempting to suggest that the apoE-HDL may be acting as a reservoir for apoE while this apoprotein plays a functional role, perhaps in remnant removal (37), in VLDL, and chylomicrons. The data of Falko et al. (13) are consistent with the hypothesis that HDL may act as a reservoir for apoE. Implicit in this hypothesis are exchange and transfer of apoE between its two major sites of residence. Our finding of a similar pattern of isoforms of apoE in VLDL and HDL from a single normal volunteer is consistent with such exchange. The absence of apoE in chylomicrons of intestinal lymph and its presence in chylomicrons isolated from blood suggest that such exchange does occur $(38,39)$. Experiments designed to test this hypothesis directly are currently in progress.

\section{ACKNOWLEDGMENTS}

The authors are grateful to Dr. DeWitt S. Goodman for his continuing encouragement and many helpful discussions. The authors thank Dr. Stephen Birken for performing the amino acid analyses. The authors wish to thank Mr. Andrey Shaw for expert and enthusiastic technical assistance and $\mathrm{Mr}$. Leonard Norbert for preparation of the manuscript.

This work was supported by grant HL 21006 from the National Heart, Lung, and Blood Institute.

\section{REFERENCES}

1. Shore, V. G., and B. Shore. 1973. Heterogeneity of plasma very low density lipoproteins. Separation of species differing in protein components. Biochemistry. 12: 502-507.

2. Shelburne, F. A., and S. H. Quarfordt. 1974. A new apoprotein of human plasma very low density lipoproteins. J. Biol. Chem. 249: 1428-1433.

3. Havel, R. J., and J. P. Kane. 1973. Primary dysbetalipoproteinemia: predominance of a specific apoprotein species in triglyceride-rich lipoproteins. Proc. Natl. Acad. Sci. U. S. A. 70: 2015-2019.

4. Utermann, G. 1975. Isolation and partial characterization of an arginine-rich apolipoprotein from human plasma very low density lipoprotiens: apolipoprotein E. HoppeSeyler's Z. Physiol. Chem. 356: 1113-1121.

5. Innerarity, T. L., R. E. Pitas, and R. W. Mahley. 1979. Binding of arginine-rich apoprotein after recombination 
with phospholipid vesicles to the low density lipoprotein receptors of fibroblasts. J. Biol. Chem. 254: 4186-4190.

6. Bersot, T. P., R. W. Mahley, M. S. Brown, and J. L. Goldstein. 1976. Interaction of swine lipoproteins with the low density lipoprotein receptor in human fibroblasts. J. Biol. Chem. 251: 2395-2398.

7. Mahley, R. W., K. H. Weisgraber, and T. Innerarity. 1976. Atherogenic hyperlipoproteinemia induced by cholesterol feeding in the Patas monkey. Biochemistry. 15: 29792985.

8. Mahley, R. W., and K. H. Weisgraber. 1974. Canine lipoproteins in atherosclerosis. II. Characterization of the plasma lipoproteins associated with atherogenic and nonatherogenic hyperlipidemia. Circ. Res. 35: 722-733.

9. Mahley, R. W., K. Weisgraber, T. Innerarity, and H. B. Brewer. 1975. Swine lipoproteins and atherosclerosis. Changes in the plasma lipoproteins and apoproteins induced by cholesterol feeding. Biochemistry. 14: 28172823.

10. Mahley, R. W., T. L. Innerarity, T. P. Bersot, A. Lipson, and S. Margolis. 1978. Alterations in human high density lipoproteins, with or without increased plasma cholesterol, induced by diets high in cholesterol. Lancet. II: 807-809.

11. Kushwaha, R. S., W. R. Hazzard, P. W. Wahl, and J. J. Hoover. 1977. Type III hyperlipoproteinemia: diagnosis in whole plasma by apolipoprotein-E immunoassay. Ann. Intern. Med. 87: 509-516.

12. Curry, M. D., W. J. McConathy, P. Alaupovic, J. H. Ledford, and M. Popovic. 1976. Determination of human apolipoprotein $\mathrm{E}$ by electroimmunoassay. Biochim. Biophys. Acta. 439: 413-425.

13. Falko, J. M., G. Schonfeld, J. L. Witztum, J. B. Kolar, S. W. Weidman, and R. Steelman. 1980. Effects on diet on apolipoprotein $\mathrm{E}$ levels and on the apoprotein $\mathrm{E}$ subspecies in human plasma. J. Clin. Endocrinol. Metab. 50: 521528.

14. Kane, J. P., T. Sata, R. L., Hamilton, and R. J. Havel. 1975. Apoprotein composition of very low density lipoproteins of human serum. J. Clin. Invest. 56: 1622-1634.

15. Blum, C. B., and L. Aron. 1979. Radioimmunoassay of human apolipoprotein E: HDL as the major locus of apoE in normal plasma. Circulation. 60(II): 119.

16. Havel, R. J., H. A. Eder, and J. H. Bragdon. 1955. The distribution and chemical composition of ultracentrifugally separated lipoproteins in human serum. J. Clin. Invest. 34: 1345-1353.

17. Blum, C. B., R. I. Levy, S. Eisenberg, M. Hall, III, R. H. Goebel, and M. Berman. 1977. High density lipoprotein metabolism in man. J. Clin. Invest. 60: 795-807.

18. Shelburne, F. A., and S. H. Quarfordt. 1977. The interaction of heparin with an apoprotein of human very low density lipoprotein. J. Clin. Invest. 60: 944-950.

19. Iverius, P. H. 1971. Coupling of glycosaminoglycans to agarose beads (Sepharose 4B). Biochem. J. 124: 677-683.

20. Greenwood, F. C., W. M. Hunter, and J. S. Glover. 1963. The preparation of ${ }^{131}$ I-labelled human growth hormone of high specific radioactivity. Biochem. J. 89: 114-123.

21. Fainaru, M., R. J. Havel, and K. Imaizumi. 1977. Radioimmunoassay of arginine-rich apolipoprotein of rat serum. Biochim. Biophys. Acta. 490: 144-155.

22. Albers, J. J., and M. C. Cheung. 1979. Radial immunodiffusion assay of lipoproteins and apoproteins: applications to high density lipoproteins. In Report of the High Density Lipoprotein Methodology Workshop. K. Lippel, editor. U. S. Dept. of Health, Education, and Welfare, Public Health Service, National Institutes of Health Publication No. (NIH) 79-1661: 251-264.
23. Lowry, O. H., N. J. Rosebrough, A. L. Farr, and R. J. Randall. 1951. Protein measurement with the Folin phenol reagent. J. Biol. Chem. 193: 265-275.

24. Rodbard, D., and D. M. Hutt. 1974. Statistical analysis of radioimmunoassays and immunoradiometric (labelled antibody) assays. In Radioimmunoassay and Related Procedures in Medicine. International Atomic Energy Agency, Vienna. 1: 165-192.

25. Dell, R. B., R. Sciacca, K. Lieberman, D. B. Case, and P. J. Cannon. 1973. A weighted least squares technique for the analysis of kinetic data and its application to the study of renal ${ }^{133}$ xenon washout in dogs and man. Circ. Res. 32: 71-84.

26. Weber, K., and M. Osborn. 1969. The reliability of molecular weight determination by dodecyl sulfate polyacrylamide gel electrophoresis. J. Biol. Chem. 244: 44064412.

27. Chiamori, N., and R. J. Henry. 1959. Study of the ferric chloride method for determination of total cholesterol and cholesterol esters. Am. J. Clin. Pathol. 31: 305-309.

28. Technicon Instruments. Total cholesterol procedure: N-24b. 1964. In Auto-Analyzer Manual. Chauncy, New York. 345.

29. Lipid Research Clinics Program. 1974. National Institutes of Health, Department of Health, Education and Welfare Publication No. (NIH) 75-628: 74-81.

30. Warnick, G. R., C. Mayfield, J. J. Albers, and W. R. Hazzard. 1979. Gel isoelectric focusing method for specific diagnosis of familial hyperlipoproteinemia type 3. Clin. Chem. 25: 279-284.

31. Spackman, D. H., W. H. Stein, and S. Moore. 1958. Automatic recording apparatus for use in the chromatography of amino acids. Anal. Chem. 30: 1190-1206.

32. Friedewald, W. T., R. I. Levy, and D. S. Fredrickson. 1972. Estimation of plasma low density lipoprotein concentration without use of the preparative ultracentrifuge. Clin. Chem. 18: 499-502.

33. Jackson, R. L., F. D. Morrisett, and A. M. Gotto, Jr. 1976. Lipoprotein structure and metabolism. Physiol. Rev. 56: 259-316.

34. Herbert, P. N., T. Forte, R. S. Shulman, M. J. LaPiana, E. L. Gong, R. I. Levy, D. S. Frederickson, and A. V. Nichols. 1975. Structural and compositional changes attending the ultracentrifugation of very low density lipoproteins. Prep. Biochem. 5: 93-129.

35. Mahley, R. W., and K. S. Holcombe. 1977. Alterations of the plasma lipoproteins and apoproteins following cholesterol feeding in the rat. J. Lipid Res. 18: 314-324.

36. Cheung, M. C., and J. J. Albers. 1979. Distribution of cholesterol and apolipoprotein A-I and A-II in human high density lipoprotein subfractions separated by $\mathrm{CsCl}$ equilibrium gradient centrifugation: evidence for HDL subpopulations with differing A-I/A-II molar ratios. J. Lipid Res. 20: 200-207.

37. Sherill, B. C., T. L. Innerarity, and R. W. Mahley. 1980. Rapid hepatic clearance of apoE $\mathrm{HDL}_{c}$ by a high-affinity receptor: identity with the chylomicron remnant transport process. J. Biol. Chem. 255: 1804-1807.

38. Green, P. H. R., R. M. Glickman, C. D. Saudek, C. B. Blum, and A. R. Tall. 1979. Human intestinal lipoproteins: studies in chyluric subjects. J. Clin. Invest. 64: 233-242.

39. Imaizumi, K., M. Fainaru, and R. J. Havel. 1978. Composition of proteins of mesenteric lymph chylomicrons in the rat and alterations produced upon exposure of chylomicrons to blood serum and serum proteins. J. Lipid Res. 19: $712-722$. 